\title{
Neumonitis secundaria a metotrexate
}

\author{
D. SÁENZ ABAD, F. J. RUIZ-RUIZ, S. MONÓN BALLARÍN, J. MOZOTA DUARTE, \\ A. MARQUINA BARCOS
}

\author{
Servicio de Urgencias. Hospital Clínico Universitario Lozano Blesa. Zaragoza
}

\author{
PNEUMONITIS ASSOCIATED TO METHOTREXATE
}

\begin{abstract}
RESUMEN
El metotrexate (MTX) es un antagonista del ácido fólico que inhibe la reproducción celular. El MTX es actualmente utilizado como antiinflamatorio en diferentes procesos. La neumonitis intersticial aguda es el principal efecto secundario pulmonar que ocurre en pacientes tratados con MTX. Describimos el caso de una neumonitis asociada a MTX en una mujer de 56 años con trombopenia autoinmune, que presentó de forma subaguda tos no productiva, disnea de reposo, fiebre y afectación del estado general. En la radiografía de tórax se observaba una afectación intersticial bilateral difusa e infiltración alveolar. Se descartó una causa infecciosa del cuadro y ante la sospecha de neumonitis asociada a MTX, se procedió a su retirada. Se administró metilprednisolona, observándose una mejoría progresiva. Tras ocho semanas las alteraciones clínicas y radiológicas habían desparecido.
\end{abstract}

PALABRAS CLAVE: Neumonitis. Metotrexate. Toxicidad pulmonary.

\begin{abstract}
Methotrexate (MTX) is a folic acid antagonist that inhibits cellular reproduction. MTX has been shown to be an effective anti-inflammatory agent. Acute interstitial pneumonitis is the main pulmonary side effect during MTX treatment. We report a case of MTX pneumonitis in a 56-year old woman with autoimmune thrombocytopenia who presented with subacute nonproductive cough, dyspnea at rest, fever, and malaise. Chest roentgenogram demonstrated bilateral diffuse interstitial and alveolar infiltration. Infectious diseases were ruled out and methotrexte-induced pneumonitis was suspected. MTX was discontinuated and methylprednisolone was prescribed. Patient improved progressively. After eight weeks, radiologic abnormalities and symptoms had disappeared.
\end{abstract}

KEY WORDS: Pneumonitis. Methotrexate. Lung toxicity.

Sáenz Abad D, Ruiz-Ruiz FJ, Monzón Ballarín S, Mozota Duarte J, Marquina Barcos A. Neumonitis secundaria a metotrexate. An Med Interna (Madrid) 2008; 25: 27-30.

\section{INTRODUCCIÓN}

El metotrexate es un fármaco utilizado en numerosas enfermedades neoplásicas, autoinmunes e inflamatorias por su efecto inmunomodulador, antiinflamatorio y antiproliferativo. Entre el 60 y $93 \%$ de pacientes tratados con metotrexate, según las series consultadas, desarrollan alguna alteración en relación con el tratamiento. La mayoría de ellas relacionadas con el gran número de efectos secundarios que presenta y que, en general, no precisan de la retirada del fármaco. De todas ellas, la neumonitis por metotrexate es el efecto adverso que con mayor frecuencia obliga a su retirada $(1,2)$. Presentamos el caso de una paciente que recibiendo tratamiento, con metotrexate, desarrollo un cuadro de pneumonitis secundaria. Así mismo realizamos una breve discusión y revisión bibliográfica de las alteraciones pulmonares secundarias al empleo de metotrexate.

\section{CASO APORTADO}

Paciente de 56 años, sin alergias conocidas ni hábitos tóxicos, que presenta como único antecedente patológico una trombopenia autoinmune diagnosticada 18 meses antes y por la cual recibía tratamiento en otro Centro. Inicialmente había recibido tratamiento con corticoides, sin éxito, y posteriormente con azatioprina que no toleró. Ante el fracaso terapéutico inicial, se le prescribió tratamiento con metotrexate a dosis de $7,5 \mathrm{mg}$ semanales administrados por vía intramuscular, que había comenzado 8 meses antes del ingreso. La paciente acude a nuestro Hospital por presentar en las últimas tres semanas disnea progresiva que llega a ser de mínimos esfuerzos, haciéndose de reposo en los últimos días. En las 72 horas previas al ingreso, la paciente refería fiebre , tos no productiva y afectación del estado general. A la exploración física la paciente presentaba taquipnea a 30 respiraciones por minuto y fiebre de $39^{\circ}$. Su saturación de oxígeno basal mediante pulsioximetría era del $82 \%$. Presentaba una tensión arterial de 120/60 mmHg. Se objetivaron lesiones cutáneas eritematosas descamativas en axila

Trabajo aceptado: 30 de agosto de 2007 
izquierda y región inguinal del mismo lado de distribución no metamérica, no pruriginosas ni dolorosas. No presentaba aumento de presión venosa yugular a $45^{\circ}$ ni adenopatías palpables. La auscultación cardiaca era rítmica, a 100 latidos por minuto, sin soplos. A la auscultación pulmonar destacaba la existencia de crepitantes secos en ambas bases. El resto de la exploración, incluyendo la neurológica, no mostraba alteraciones.

Al ingreso lo más destacado en las exploraciones complementarias solicitadas fue una elevación en las cifras de LDH: 1196 U/l, sin alteraciones significativas en el resto de parámetros bioquímicos estudiados. Presentaba una discreta tombopenia (114.000 plaquetas por $\mathrm{mm}^{3}$ ). El resto de valores del hemograma se encontraban en rango de normalidad. Los valores de la gasometría arterial eran: $\mathrm{pH}$ : 7,42, $\mathrm{pO}_{2}$ : $45 \mathrm{mmHg}$, $\mathrm{pCO}_{2}: 30 \mathrm{mmHg}$, saturación de $\mathrm{O}_{2}: 82 \%$, bicarbonato: 19 $\mathrm{mmmol} / \mathrm{l}$. El ECG mostraba una taquicardia sinusal, sin otros hallazgos. En la radiografía de tórax se observaba un patrón intersticial bilateral en bases y campos medios (Fig. 1).

Ante la sospecha de neumonitis por metotrexate, se procedió a la retirada del fármaco en el momento del ingreso. Se descartó una etiología infecciosa del cuadro, resultando negativos los cultivos de sangre, orina y esputo. La determinación de los antígenos en orina

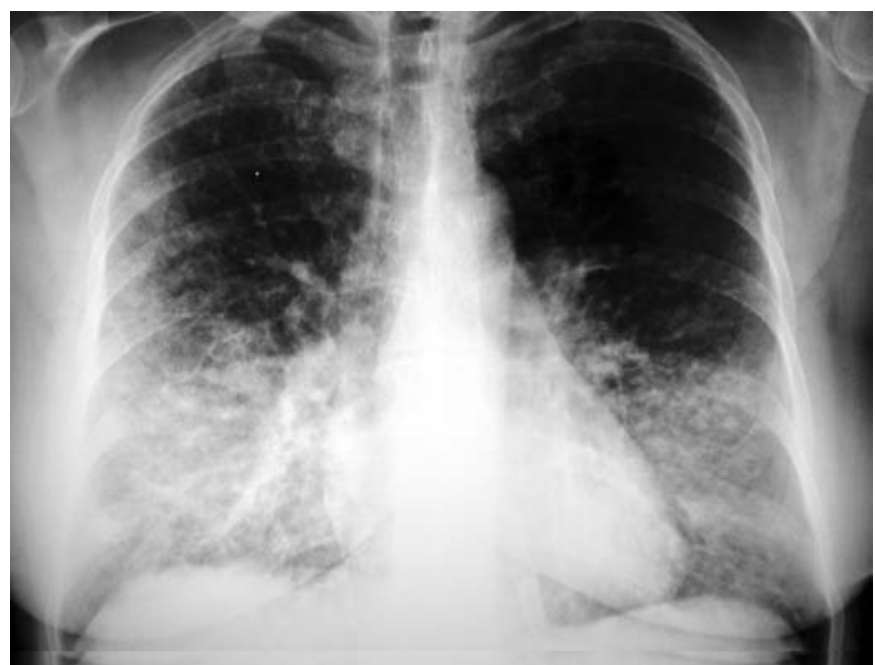

Fig. 1. de Legionella y neumococo fue negativa. Se solicitaron serologías para neumonías atípicas y virus que también resultaron negativas La investigación para Mycobacterium tuberculosis resultó negativa. La batería de autoanticuerpos realizada no apoyo el diagnóstico de patología de naturaleza autoinmune. Se solicito una TAC de tórax que mostró engrosamiento de septos intersticiales interlobulares de forma difusa y bilateral con presencia de nodulillos y un patrón en vidrio deslustrado con distribución bilateral y simétrica (Fig. 2A). A pesar de mantener una adecuada mecánica ventilatoria, la paciente precisaba altos flujos de oxígeno para conseguir una oxemia mayor del $90 \%$. Dada la importante afectación clínica se añadió al tratamiento de soporte (antitérmicos, fluidoterapia y oxígeno a alto flujo) metilprednisolona a dosis de $1 \mathrm{mg} / \mathrm{kg} / \mathrm{d}$ áa. La paciente evolucionó satisfactoriamente desapareciendo la fiebre y el resto de síntomas. Al alta presentaba una gasometría con los siguientes valores: $\mathrm{pH}: 7,42, \mathrm{pO}_{2}: 71, \mathrm{pCO}_{2}$ : 44, bicarbonato: 28 , saturación de oxígeno: 94\%. En una TC de control, realizada 8 semanas después del alta se observó la normalización de las alteraciones presentes al ingreso (Fig. 2B).

\section{DISCUSIÓN}

El metotrexate es un fármaco citotóxico antimetabolito que inhibe la reproducción celular al actuar como antagonista del ácido fólico. Inicialmente utilizado como fármaco antineoplásico, en el momento actual sus principales indicaciones se centran en procesos de naturaleza autoinmunitaria, destacando su uso en artritis reumatoide (3). Es por ello que la mayoría de casos y estudios publicados de toxicidad hacen referencia a pacientes con artritis reumatoide. En el caso presentado, se utilizó como tratamiento de una trombopenia autoinmune, no pudiendo aportar más datos al respecto al haber sido tratada en otro centro. Si bien no constituye un tratamiento estandarizado en este cuadro, se han descrito casos de utilización del fármaco en esta patología (4)

Se estima que su incidencia y prevalencia es del $3.9 \%$ y $5.5 \%$ de cada 100 pacientes/año expuestos a metotrexate, respectivamente. Actualmente se considera una reacción inmune idisincrática más que un proceso relacionado con la dosis empleada. Las variables con mayor frecuencia relacionada
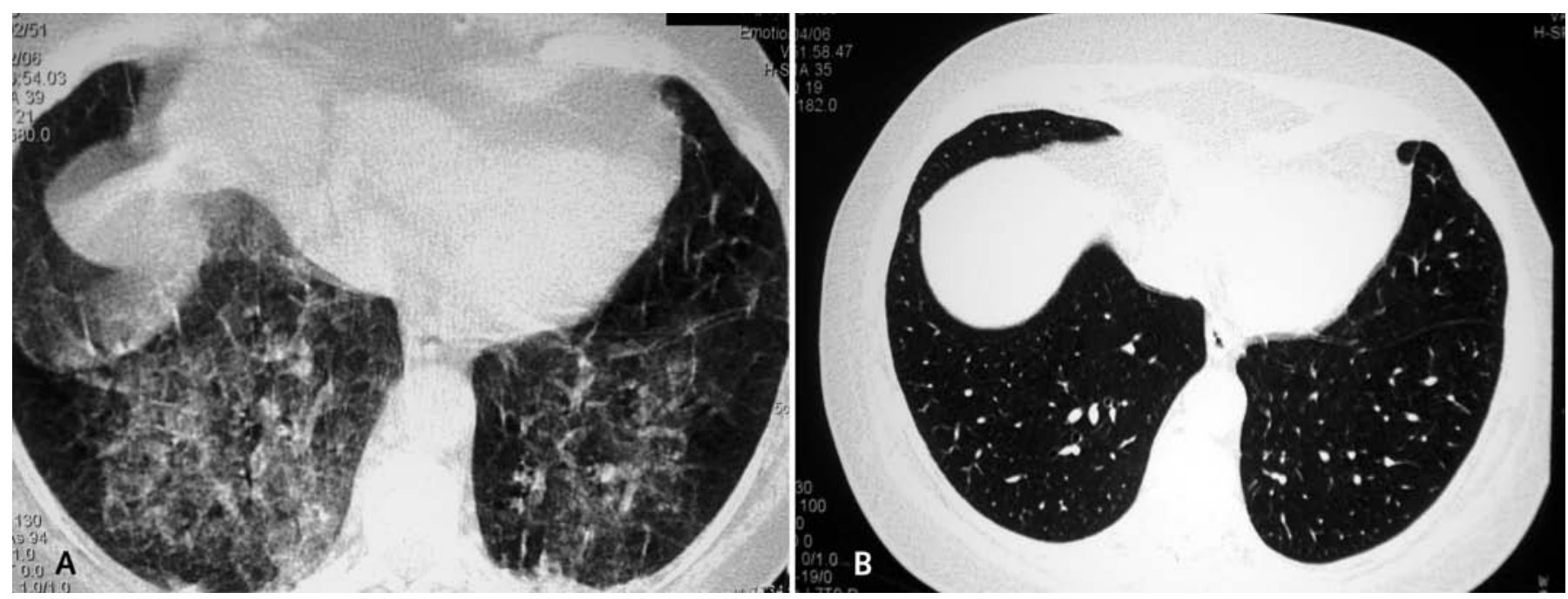

Fig. 2. 
con la neumonitis han sido la edad y el uso previo de fármacos modificadores de la artritis reumatoide. Otras variables que también se han sugerido son: afectación pulmonar por artritis reumatoide, hipoalbuminemia, diabetes mellitus, administración diaria del fármaco en vez de semanal, patología pulmonar previa, alteración de las pruebas de función respiratoria previa al tratamiento, utilización concomitante de otros fármacos (como ácido acetilsalicílico u antiinflamatorios no esteroideos, penicilina, fenitoína, ...) y disminución de la eliminación del fármaco (debido a insuficiencia renal, ascitis...) (5-7). Las alteraciones pulmonares observadas en pacientes que reciben tratamiento con metotrexate pueden ser infecciosas, inflamatorias y posiblemente neoplásicas aunque este último mecanismo de producción es desconocido. Se ha sugerido que el tratamiento mantenido con metotrexate puede favorecer la proliferación de linfomas aunque esto no está totalmente demostrado (8). Entre las causas infecciosas, la inmunosupresión que provoca, aumenta el riesgo de infecciones oportunistas, siendo la más frecuente la producida por Pneumocystis jiroveci (9).

Dentro de las alteraciones con componente inflamatorio, la neumonitis por hipersensibilidad es la forma más frecuente de toxicidad pulmonar asociada al uso de metotrexate. Otros procesos, menos frecuentes, de características inflamatorias descritos en relación con el tratamiento con metotrexate son la bronquiolitis obliterante con neumonía organizada, edema pulmonar no cardiogénico, fibrosis pulmonar e hiperreactividad bronquial. El mecanismo patogénico por el que se produce el daño pulmonar no ha sido aclarado, habiéndose sugerido la posibilidad de daño directo del metotrexate o bien a través de una reacción de hipersensibilidad $(10,11)$.

La forma de presentación puede ser aguda, subaguda o crónica. De todas ellas la forma más frecuente es la subaguda en la que la toxicidad se desarrolla en el primer año de tratamiento. Cursa con disnea, tos no productiva, fiebre, cianosis y evolución a fibrosis en el $10 \%$ de los casos. Un $20 \%$ de los pacientes presentan manifestaciones cutáneas y hasta el $50 \%$ eosinofilia periférica. La forma aguda aparece hasta en el 5\% de pacientes tratados por artritis reumatoide y cursa con síntomas inespecíficos, tanto respiratorios como sistémicos, que progresan durante semanas, pudiendo evolucionar a un fallo respiratorio rápidamente (12).

No existe ninguna prueba diagnóstica definitiva por lo que el diagnóstico requiere un alto índice de sospecha y apoyarse en la clínica, radiología y resultados obtenidos tras la retirada del fármaco. Ocasionalmente puede ser de utilidad los datos obtenidos del lavado broncoalveolar (LBA) y biopsia pulmonar. La alteración radiológica más característica es la afectación intersticial difusa. En algunos pacientes este patrón evoluciona a un infiltrado alveolar parcheado y lesiones nodulares. En una minoría de casos pueden observarse otras alteraciones radiológicas (adenopatías hiliares, atelectasias, derrame pleural,...). Es excepcional observar una radiografía tórax normal. La tomografía de alta resolución (TACAR) es más sensible que la radiología simple para detectar la afectación intersticial. La extensión de las lesiones en la TACAR se correlaciona con la disminución de los volúmenes pulmonares en las pruebas de función respiratoria (PFR). Un patrón restrictivo con disminución de la capacidad de difusión del CO (DLCO) es el hallazgo más frecuente observado en las PFR de estos pacientes $(13,14)$.
Tanto los resultados obtenidos en el LBA como en la biopsia pulmonar son inespecíficos y pueden estar presentes en otros mucho procesos de naturaleza inflamatoria. Los hallazgos observados en la biopsia pulmonar son alveolitis con hiperplasia de células epiteliales, infiltrado de eosinófilos, pequeños granulomas y fibrosis extensa con áreas en panal de abeja de predominio en bases. Ninguna de estas dos exploraciones complementarias son obligatorias para establecer el diagnóstico de neumonitis por metotrexate. Searles y McKendry propusieron unos criterios para el diagnóstico de la toxicidad pulmonar por metotrexate cuya utilidad clínica debe individualizarse (Tabla I). El diagnóstico se considera definitivo si 6 o más criterios están presentes, probable si reúne 5 y posible si son 4 los criterios (15). En el caso presentado el diagnóstico vino dado por la alta sospecha clínica y avalado por los criterios de Searles y McKendry. Nuestra paciente reunía 7 de los 9 criterios propuestos (todos, excepto los criterios número 6 y 9 de la tabla I).

\section{TABLA I}

CRITERIOS DE SEARLES Y MCKENDRY PARA EL DIAGNÓSTIVO CLIINICO DE NEUMONITIS SECUNDARIA A METOTREXATE

1. Disnea de menos de 8 semanas de evolución

2. Taquipnea mayor a 28 respiraciones por minutos y tos no productiva

3. Fiebre mayor de $38^{\circ}$

4. Saturación de oxígeno menor de $90 \%$ respirando aire ambiente el inicio del cuadro

5. Leucocitos en sangre menor de 15000 por $\mathrm{mm}^{3}$

6. Patrón restrictivo en las pruebas de función respiratoria y capacidad de difusión disminuida (DLCO menor de $70 \%$ del valor previsto para la edad)

7. Hemocultivos y cultivos de esputo negativos (este criterio es de obligado cumplimiento)

8. Afectación pulmonar intersticial o infiltrados alveolares en la radiología

9. Neumonitis por hipersensibilidad en la histopatología sin evidencia de organismos patógenos

Respecto al tratamiento es imprescindible descartar causas infecciosas. Así ante la sospecha de enfermedad infecciosa esta indicada la terapia antimicrobiana empírica una vez extraídas muestras biológicas para cultivo. El primer paso en el tratamiento ante la sospecha de neumonitis por metotrexate consiste en la retirada de dicho fármaco tan pronto como sea posible. En la mayoría de casos, esto provoca una mejoría clínica en los siguientes días, seguida de una mejoría radiológica a lo largo de semanas. El tratamiento con corticoides no esta avalado por estudios que apoyen su uso, si bien se han descrito casos en que se han empleado con éxito. Su uso podría estar justificado en caso de afectación clínica muy importante o en caso de persistir alteraciones clínicas o radiológicas a pesar de retirar el metotrexate. Se recomienda comenzar con metilprednisolona a dosis de $1 \mathrm{mg} / \mathrm{kg}$ peso/día (16). En aquellos casos con mala respuesta se ha descrito mejoría de la función pulmonar tras tratamiento con ciclofosfamida. La mortalidad del cuadro se sitúa en el 1\%. En la mayoría de casos la recuperación es completa, si bien un por- 
centaje variable de pacientes pude mostrar secuelas radiológicas o en las pruebas de función respiratoria $(6,17)$. La administración de ácido fólico, utilizada de forma concomitante junto al metotrexate para atenuar o evitar los síntomas gastrointestinales, las alteraciones hematológicas o de las pruebas de función hepática, no ha demostrado su efectividad en la prevención de la toxicidad pulmonar, si bien se ha objetivado cierta eficacia del acido folínico $(18,19)$.

\section{Bibliografía}

1. Gauhar UA, Gaffo AL, Alarcon GS. Pulmonary manifestations of rheumatoid arthritis. Semin Respir Crit Care Med 2007; 96: 1641-5

2. Grove ML, Hassell AB, Hay EM, Shadforth MF. Adverse reactions to disease-modifying anti-rheumatic drugs in clinical practice. Q J Med 2001; 94: 309-19.

3. Swierkot J, Szechinski J. Methotrexate in rheumatoid arthritis. Pharmacol Rep 2006; 58: 473-92

4. Llamas P, Busto M J, Diez JL, Cabrera R, Regidor C, Fores R, et al. Eficacia de distintos tratamientos en el manejo de la púrpura trombocitopénica idiopática (PTI) del adulto. Sangre 1995; 40: 181-85

5. Alarcon, GS, Kremer, JM, Macaluso, M, Weinblatt ME, Cannon GW, Palmer WR, et al. Risk factors for methotrexate-induced lung injury in patients with rheumatoid arthritis: A multicenter, case-control study. Ann Intern Med 1997; 127:356-64

6. Imokawa S, Colby T.V, Leslie K.O, Helmers R.A. Methotrexate pneumonitis: review of the literature and histopathological findings in nine patients. Eur Respir J 2000; 15: 373-81

7. Saravanan V, Kelly C. A. Reducing the risk of methotrexate pneumonitis in rheumatoid arthritis. Rheumatology 2004;43:143-47

8. Jardine DL, Colls BM. Hodgkin's disease following methotrexate therapy for rheumatoid arthritis. N Z Med J 2002; 115: 293-4

9. Kaneko Y, Suwa A, Ikeda Y, Hirakata M. Pneumocystis jiroveci pneumonia associated with low-dose methotrexate treatment for rheumatoid arthritis: report of two cases and review of the literature. Mod Rheumatol 2006; 16: 36-38

10. Cho I, Mori S, Imamura F, Kiyofuji C, Sugimoto M. Methotrexate pneumonia lacking dyspnea and radiographic interstitial patterns during treatment for early rheumatoid arthritis: bronchoalveolar lavage and transbronchial lung biopsy in a differential diagnosis. Mod Rheumatol 2007; 17: 256-61
11. Barrera P, Laan RF, von-Riel Pl, Dekhuijzen PN, Boerbooms AM, van de Putte LB. Metotrexate-related pulmonary complication in rheumatoid arthritis. Ann Rheum Dis 1994; 53: 434-39

12. Kremer Jm, Alarcon GS, Weinblatt ME, Kaymakcian MV, Macaluso M, Cannon GW, et al. Clinical, laboratory, radiographic, and histopathologic features of methotrexate-associated lung injury in patients with rheumatoid arthritis: a multicenter study with literature review. Arthritis Rheum 1997; 40: 1829-37

13. Rossi SE, Erasmus J.J, McAdams H.P, Sporn T.A, Goodman P.C. Pulmonary drug toxicity: radiologic and pathologic manifestations. Radiographics 2000; 20:1245-59

14. Arakawa H, Yamasaki M, Kurihara Y, Yamada H, Nakajima Y. Methotrexate-induced pulmonary injury: serial CT findings. J Thorac Imaging 2003; 18: 231-6

15. Searles G, McKendry RJR. Methotrexate penumonitis in rheumatoid arthritis: potential risk factors. Four cases and review of the literature. J Rheumatol 1987; 14: 1164-1171.

16. Balk RA. Methotrexate-induced lung injury. In: UpToDate, Rose BD (Ed), UpToDate, Waltham, USA, 2006

17. Suwa A, Hirakata M, Satoh S, Mimori T, Utsumi K, Inada S. Rheumatoid arthritis associated with methotrexate-induced pneumonitis: improvement with intravenous cyclophosphamide therapy. Clin Exp Rheumatol 1999; $17: 335-38$

18. Morgan SL, Oster RA, Lee JY, Alarcon GS, Baggott JE. The effect of folic acid and folinic acid supplements on purine metabolism in methotrexate-treated rheumatoid arthritis. Arthritis Rheum 2004; 50 3104-11

19. Whittle S. L, Hughes R. A. Folate supplementation and methotrexate treatment in rheumatoid arthritis: A review. Rheumatology 2004; 43: 267-71 